\title{
Histologically Measured Cardiomyocyte Hypertrophy Correlates with Body Height as Strongly as with Body Mass Index
}

\author{
Richard E. Tracy ${ }^{1}$ and Gary E. Sander ${ }^{2}$ \\ ${ }^{1}$ Department of Pathology, Louisiana State University Health Sciences Center, 1901 Perdido Street, P5-1, \\ New Orleans, LA 70112, USA \\ ${ }^{2}$ Tulane University Heart and Vascular Institute, Tulane University 1430 Tulane Avenue SL-48, \\ New Orleans, LA 70112, USA \\ Correspondence should be addressed to Richard E. Tracy, rtracy@lsuhsc.edu
}

Received 19 January 2011; Revised 15 March 2011; Accepted 17 March 2011

Academic Editor: Lars S. Maier

Copyright ( $) 2011$ R. E. Tracy and G. E. Sander. This is an open access article distributed under the Creative Commons Attribution License, which permits unrestricted use, distribution, and reproduction in any medium, provided the original work is properly cited.

Cardiac myocytes are presumed to enlarge with left ventricular hypertrophy (LVH). This study correlates histologically measured myocytes with lean and fat body mass. Cases of LVH without coronary heart disease and normal controls came from forensic autopsies. The cross-sectional widths of myocytes in H\&E-stained paraffin sections followed log normal distributions almost to perfection in all 104 specimens, with constant coefficient of variation across the full range of ventricular weight, as expected if myocytes of all sizes contribute proportionately to hypertrophy. Myocyte sizes increased with height. By regression analysis, height $^{2.7}$ as a proxy for lean body mass and body mass index (BMI) as a proxy for fat body mass, exerted equal effects in the multiple correlation with myocyte volume, and the equation rejected race and sex. In summary, myocyte sizes, as indexes of LVH, suggest that lean and fat body mass may contribute equally.

\section{Introduction}

Early reports concerning myocyte size in the human left ventricle, relying heavily upon autopsy specimens [1-3], often found that [4] "the individual contractile muscle cells $\ldots$ are roughly cylindrical ... measuring 17 to $25 \mu \mathrm{m}$ in diameter and 60 to $140 \mu \mathrm{m}$ in length," giving length to diameter ratio of about 5 to 1 . Recent studies, relying on enzymatic isolation of myocytes into suspension from surgical specimens [5-14], commonly model myocytes as elliptic cylinders of variable eccentricities and length to width ratios under assorted conditions [5-7]. Variations between these models, arising from disparate methodologies, call for further inquiry to resolve their causes. Cross-sectional profiles of myocytes, whether isolated or in sections, display a daunting irregularity of outline, especially of major axis, although less so of the minor axis. Measurements of minor axis are therefore emphasized in this report of findings with $\mathrm{H} \& \mathrm{E}-$ stained paraffin sections of forensic autopsy specimens.
Left ventricular mass increases in many hyperergopathic states, often culminating in systolic dysfunction $[15,16]$. Behavior by the full range of myocyte sizes, and not just their average, merits attention. Several authors [7-10] have reported unimodal distribution curves of myocyte sizes, consistently showing conspicuous upward skewness. These authors did not report statistical curve fitting; however, graphic inspection raised the possibility of log normal fits, which could imply that the smallest and the largest myocytes may all respond alike [7-9], a possibility seldom discussed. Grant et al. [17] stated a rarely encountered suggestion that "eccentric hypertrophy is analogous to normal growth, the process which converts a neonatal left ventricle into an adult chamber." It is therefore easy to envision that the process could become exaggerated by uncommon growth into the tallest statures, which could be viewed as a form of eccentric hypertrophy that is progressing toward pathological thresholds. 
Myocyte breadth clearly increases in company with concentric hypertrophy irrespective of etiology $[1-9,12,13]$. However, the behavior of myocyte breadth as the chamber dilates is controversial $[5,6,12,13,17]$. Data in the present report offer some comment upon this question.

Assessment of myocyte volume is problematic at best in the data reported here. However, some rough estimates applicable to group averages can be explored. This is done here for only one limited purpose, to relate myocyte sizes to body weight.

This study in a series of 104 forensic autopsies included all encountered instances of cardiomegaly (excluding coronary heart disease), instances that were often later attributed to hypertension, obesity, or idiopathic cardiomyopathy (46 cases), aortic stenosis (4), mitral deformity (1), or cor pulmonale (2). Techniques for measuring myocyte sizes were developed and applied to this series of specimens.

\section{Methods}

2.1. Subjects. Between November 2007 and May 2009, the Orleans Parish Coroner's Office supplied 104 specimens. All cases with cardiomegaly (whole heart weight $>399 \mathrm{gm}$ in women and $>449 \mathrm{gm}$ in men), other than coronary heart disease, were included. Death was attributed to cardiovascular causes in 42 cases and to noncardiovascular causes in 62 cases (with 46 instances of violence or drugs). Left ventricular hypertrophy (LVH) occurred in 33 of the 42 cardiovascular cases and 20 of the 62 noncardiovascular cases (many chosen because of the presence of incidental LVH). Noncardiovascular cases were chosen to have $<12$ hours postmortem interval, no immediately preceding hospitalization, and cause of death unrelated to cardiovascular disease. The 42 cases classed as cardiovascular deaths included chronic renal failure $(N=1)$, cerebrovascular disorders (3), aortic stenosis (4), mitral deformity (1), pulmonary conditions (8, cor pulmonale, pulmonary embolus, arrhythmogenic right ventricular dysplasia), dilated hypertrophy (23), and LVH without dilatation (2). Hypertrophic cardiomyopathy, as defined by myocyte disarray [18], was not encountered. Clinical information, most notably on hypertension, was not available in these forensic cases, because review of clinical records was omitted; therefore the IRB declared this autopsy study exempt from their review. Body weight was unavailable in the first 34 cases.

\subsection{Left Ventricular Weight (LVW), Hypertrophy (LVH),} Chamber Diameter (CD), and Wall Thickness (LVT). Ventricles of the fresh heart were divided from the atria, separated, and the epicardial fat dissected away. The method of Bove et al. [19] was modified by using the fresh rather than the formalin-fixed heart and by placing the trabeculae carneae and papillary muscles from the right side of the interventricular septum with the right ventricle. Data of Deveraux et al. [20] were used to calculate 219 grams in men and 199 grams in women as cutoff points for LVH. Chamber diameter (CD) was measured with a ruler on the high chordal plane (touching the edges of the opened mitral leaflets) through the left ventricle. Thickness of lateral and posterior walls was measured with a ruler and averaged to find LVT; these exclude trabeculae carneae and epicardial fat.

2.3. Preparations for Histology. A slice of myocardium presenting the high chordal plane was formalin fixed for 16 days. Samples were excised from lateral, posterior, and septal walls of the LV for sectioning both parallel and perpendicular to the plane in the first 34 cases, and thereafter only in the perpendicular plane. Paraffin-embedded samples were sectioned at $6 \mu \mathrm{m}$ and stained with H\&E. A subset of specimens measured before and after processing into slides showed mean shrinkage of $17.2 \%$ (SEM $=0.96 \%)$, agreeing with published values [21, 22]. Adjustment by $1.21=1 / 0.828$ was done upon subsequent histological measurements, although this is not needed for the relative comparisons of myocyte breadths in specimens reported here.

2.4. Myocyte Profiles. Myocyte profiles in cross-section (Figure 1(a)) were evaluated by imposing an ellipse upon each profile and measuring the minor axis of the idealized outline. In longitudinal planes of cut, diameters were measured at positions exposing substantial lengths of uniform width (Figure 1(b)). These measurements were made upon black-and-white photographic prints using a digital caliper accurate to $0.01 \mathrm{~mm}$. The first 34 cases were used to explore various sampling schemes. Thereafter, measurements were taken in 60 anucleate cross-sectioned profiles from each of 4 sites in the LV free wall.

2.5. Sampling Variations. Myocyte profiles with nucleus present were compared to profiles without visible nuclei as a ratio, and the mean ratio departed substantially from 1.0 (mean $=1.195$, SEM $=0.012, N=34$ ). In all following analyses, therefore, nucleated profiles were omitted from the samples for simplicity and consistently. The calibers measured in longitudinal profiles held a ratio to those in cross-sections of mean $=1.048($ SEM $=0.012)$; since this is significantly different from 1.0, longitudinal profiles were omitted from all but one of the following analyses. The two samples of LV free wall, posterior and lateral, yielded caliber ratios to each other of mean $=1.021($ SEM $=0.014)$, which does not differ significantly from $1.0(P=.15)$, but the ratio of caliber in the interventricular septum to that in the mean free wall did differ from 1.0 (mean $=0.944$, SEM $=0.012$, $P<.001$ ); hence the following analyses omit septal samples. Between subendocardial and subepicardial sites the caliber ratio had mean $=1.002(\mathrm{SEM}=0.009, P=.84)$, hence further analyses include sites at arbitrary depths into the ventricular wall.

2.6. Myocyte Volume (MV) and Breadth (MB). In the idealized model of a myocyte as an elliptic cylinder, its dimensions are major axis, $A$, minor axis, $B$, and length $L$, so that the volume is $\mathrm{MV}=\pi \mathrm{ABL} / 4=\pi r^{2} \mathrm{~L}$. The name "width" is often given to the quantity $2 r$ in the imaginary circle of radius $=r$. However, the name "width" is also 


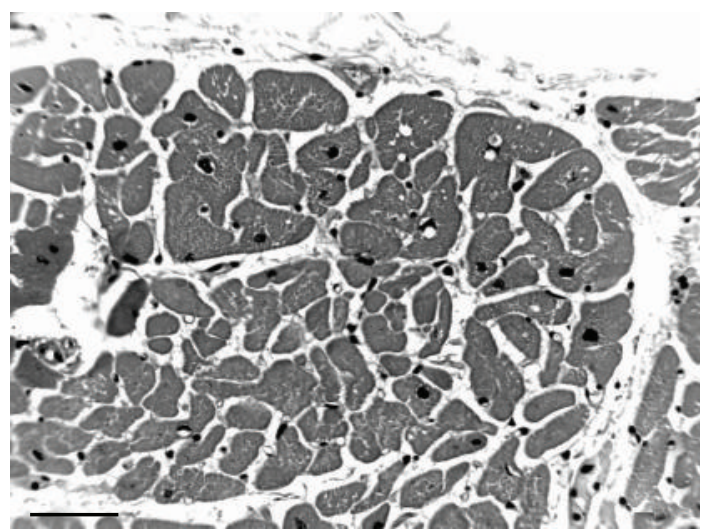

(a)

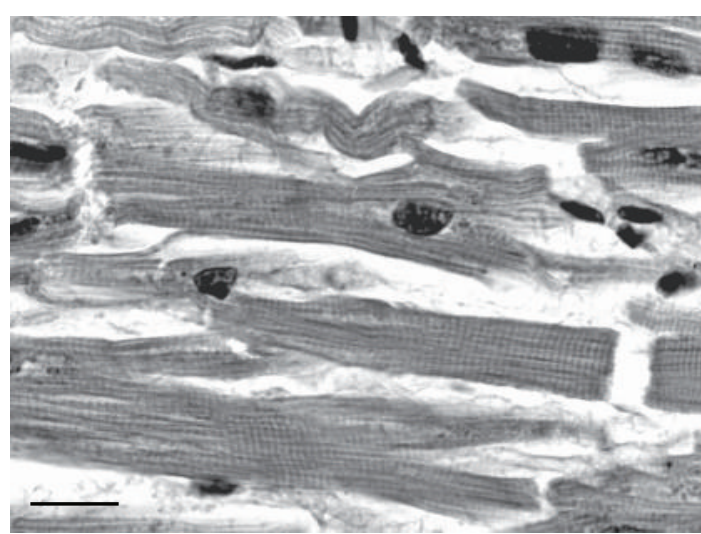

(b)

Figure 1: Cross-sectional (a) and longitudinal (b) planes of cut as assessed in the first 34 cases. H\&E, bars $=30 \mu \mathrm{m}$.

often used for the major axis or for the minor axis, making the name "width" ambiguous. To avoid this ambiguity, the name "breadth", $\mathrm{MB}=$ myocyte breadth, is assigned to the quantity $2 r$ calculated from the minor axis, as explained next.

2.7. Elliptic Eccentricity. Measurements in cross-sectioned myocytes estimate the quantity B, minor axis. Measurements on longitudinal planes of cut represent random diameters in all directions across the elliptic myocyte, and their average is a composite of these varying lengths. In the 34 specimens measured in both ways, the means of the two methods averaged a ratio of $1.048(\mathrm{SEM}=0.012)$. By a method of iteration it can be found that $\mathrm{A} / \mathrm{B}=11 / 10$ yields a close approximation to the observed ratio of longitudinal/cross calibers $=1.048$ given above. Knowing that $\mathrm{AB} / 4=r^{2}$ and that $\mathrm{A}=11 \mathrm{~B} / 10$, the area becomes $\left(\pi \mathrm{B}^{2} \times 11 / 10\right) / 4=\pi r^{2}$ and $2 \sqrt{\mathrm{B}^{2}((11 / 10) / 4)}=2 r=\mathrm{MB}=1.048 \times \mathrm{B}$ is the mean value for myocyte breadth. This is the function for determining $\mathrm{MB}$ from the measured minor axis, $\mathrm{B}$, and conversion to $\mathrm{MB}$ allows comparisons with published data.

2.8. Sarcomere Length. In a subset of 59 specimens, Zbands were counted within $30 \mu \mathrm{m}$ long segments of myocytes (Figure 1(b)). Counts divided by 30 (times 1.21 to correct for preparation shrinkage) yields the sarcomere length. This value averaged $1.68 \mu \mathrm{m}(\mathrm{SEM}=0.044 \mu \mathrm{m}, N=59)$. In a study of 6 hearts from ordinary human autopsies, Gerdes et al. [14] found a comparable average sarcomere length of $1.68 \mu \mathrm{m}$ in isolated myocytes. Hearts acquired for enzymatic isolation of myocytes typically undergo rapid fixation of fresh organs; sarcomere lengths in those organs have generally ranged around $2.0 \mu \mathrm{m}[8,11,13,14]$. The ratio $1.68 / 2.0=0.84$ is needed later as an adjustment to allow transference of published diastolic data into formulas developed here for systolic data in this study. Sarcomere lengths measured here did not correlate with LVW, renal evidence of hypertension, age, body length, body weight, or skewness of the log normal fits $(P=.15$ to .98$)$.
2.9. Myocyte Length (ML). This study gives only limited and somewhat peripheral attention to myocyte volume (MV). This quantity has three spacial dimensions and therefore seems appropriate for comparison with body weight (a three-dimensional variable and the only one here calling for some passing attention). The necessary data require only mean values for each specimen and have no need for individual measurements. Fortunately, some data offering a way to estimate these means are available in prominent publications, and this estimation is examined next: in a study of maturing spontaneously hypertensive rats from birth to 24 months, many with LVH, Tamura et al. [13] found that "... cell length should be directly reflected by proportional alterations in chamber circumference." In the rats, circumference of $20 \mathrm{~mm}$ corresponded to $\mathrm{L}=135 \mu \mathrm{m}$, indicating 148 myocytes in the circumference if they are envisioned in an imaginary arrangement end to end. A similar proportionality in the human can only be inferred indirectly from available sources. Using the data of Gerdes et al. [5], taking chamber circumference data from Teichholz et al. [23], healthy nonhypertrophied hearts had $\mathrm{L}: \mathrm{MB}=7: 1$, $\mathrm{L}=134 \mu \mathrm{m}$, and $\mathrm{CD}=52 \mathrm{~mm}$, hence a value of 1,313 can be estimated for the number of myocytes envisioned as circumferentially tandem, about 8.9 times those in the rat. For dilated ventricles, $\mathrm{L}: \mathrm{MB}=11: 1, \mathrm{~L}=203 \mu \mathrm{m}$, and $\mathrm{CD}=82 \mathrm{~mm}$, yielding 1,269 as the estimate for number of circumferentially tandem myocytes, 8.6 times that in the rat. The ratio of $\mathrm{CD}$ to $\mathrm{L}$ is nearly identical in the human ventricles of both size ranges, in keeping with the result seen in rats of near proportionality of myocyte length to chamber diameter. In the data of this study, the hearts lacking hypertrophy with mean $C D=28.0 \mathrm{~mm}$ had mean $\mathrm{MB}=17.4 \mu \mathrm{m}$. Hence a simple formula can yield approximate estimates of $\mathrm{L}$ for any particular $\mathrm{CD}$; $\mathrm{L}=$ $7 \times 0.84 \times 17.4 \times \mathrm{CD} / 28.0=3.654 \mathrm{CD} \times 10^{-3} \mu \mathrm{m}$, where the correction factor 0.84 adjusts for the systolic condition of the specimens examined here, and 17.4/28.0 is taken as the reference standard. This formula will be reconsidered in the later discussion. The values for MV determined using 
this estimate of length correlates with $\mathrm{MB}^{3}$ with $r=0.59$ $(P<.001)$.

2.10. Myocyte Numbers (MN). Total number of myocytes $(\mathrm{MN})$ in a ventricle is of limited interest in this report. For that restricted purpose it can simplistically be calculated as $\mathrm{MN}=0.75 \times \mathrm{LVW} / \mathrm{MV}$, ignoring the negligible effect of specific gravity. The term 0.75 is a commonly reported estimate [11] of myocytes as a proportion of total LVW (myocyte volume fraction). MN calculated this way fails to remain constant across the range of ventricular weights in this data set, and it is possible to correct this defect by using an adjustment factor, $j$. Although Tamura et al. [13] report their findings as favoring approximate proportionality of myocyte length to chamber circumference, the regression equation that they report actually contains a significant intercept term, showing departure from proportionality. With the help of that regression equation, (1) can be derived

$$
j=1.2 \times(\mathrm{CD}-28.0) .
$$

The adjusted myocyte length is $(3.654 \times \mathrm{CD}-j) \times 10^{-3} \mu \mathrm{m}$. Myocyte numbers, using MV calculated with this adjusted length, yields an adjusted quantity that does not correlate with LVW $(r=-0.11, P=.29)$ and is therefore constant across the range of ventricular weights; it also does not correlate with race $(P=.98)$, sex $(P=.97)$, height $(P=$ $.35)$, or body weight $(P=.60)$, demonstrating the expected constancy of myocyte numbers between these groupings. This formula will be reconsidered in the later discussion. Between MV and its adjusted value, $r=0.99$ is found, so that either can substitute for the other in correlation analyses.

2.11. Fat and Lean Body Mass Proxies. Body mass index, BMI = weight/height ${ }^{2}\left(\mathrm{~kg} / \mathrm{m}^{2}\right)$, is often used as a proxy for body fat mass in proportionate comparisons between individuals. Indexed left ventricular mass, LVW/height ${ }^{2.7}$ $\left(\mathrm{Gm} / \mathrm{m}^{2.7}\right)$, is also often used in comparisons of individuals, implying that height ${ }^{2.7}$ is a proxy for lean body mass. In this data set, the 70 cases with complete data revealed a multiple correlation of these two proxies with total body weight of $r=0.997$, indicating that they jointly capture all of the variations between individuals in total body weight, so that their use as proxies passes this test of validity. The standardized regression equation is weight $=0.347 *$ height $^{2.7}$ $+0.816 * \mathrm{BMI}$, implying that variation of fat mass between individuals is more than twice as important as variation of lean mass for determining total body weight.

2.12. Mean Arterial Pressure (MAP). Intimal thickness of interlobular arteries in the renal cortex was measured in 100 cases, and mean arterial pressure (MAP) calculated using a previously derived regression equation $[24,25]$ ( $\mathrm{MAP}=$ $(\mathrm{S}+2 \mathrm{D}) / 3)$.

2.13. Statistical Method. These are all commonly used techniques available in the SAS programs (SAS Institute,

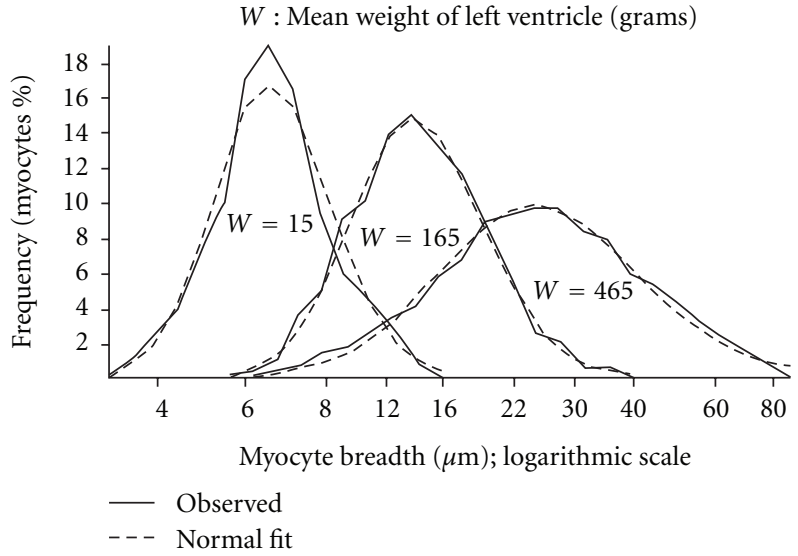

FIGURE 2: Observed frequency distributions and their log normal fitted curves for a 6 -week-old infant $(W=15)$ and two selected pools of left ventricles.

Cary NC). Some of the linear regression equations yielded insignificant intercept terms so that the appropriate equations are forced through the origin; the correlation coefficients reported with these equations are those retaining the intercept terms. For computing odds ratios in the logistic regressions, the variables were measured in units of standard deviation. For consistency and economy in testing of log normal fits to distribution curves only the Shapiro-Wilk test was applied.

\section{Results}

3.1. Tests of Log Normal Fits. Figure 2 plots log normal distribution curves for $\mathrm{MB}$ as measured in the LV of a 6week-old infant (LVW $=15 \mathrm{Gm}$ ), a pool of 6 cases of low ventricular weights, and a pool of 6 cases with high ventricular weights (mean and SD were closely alike in these cases chosen for pooling). The low weight group was chosen to illustrate a distribution that fails to reject the log normal fit ( $P>.05$ by Shapiro-Wilk test), while the high weight group exemplifies the kind of misfit that is typical of curves that significantly depart from the log normal. The log normal was rejected as misfit in 33 of the 104 cases at $P<.1$ and 53 cases at $P<.2(32 \%$ and $51 \%$ resp. in Table 1 , rather than the randomly expected $10 \%$ and $20 \%$, resp.). The skewness had a mean of $-0.140(P<.001$ for difference from zero, $\mathrm{SD}=0.211)$, and was below zero in 73 specimens $(70 \%)$. Of the 33 seriously misfit cases, 22 displayed skewness < -0.140 (correlation of skewness with $P$-values for the misfit was $r=0.38, P<.001$ in the 104 cases). The 56 cases with skewness $>-.139$ had $P$-values for the log normal fits which appear acceptably random $(.1>P>.2$ by chi-square test in Table 1). Correlation of skewness with LVW was not significant $(P=.16)$. The coefficient of variation $(\mathrm{CV})$ of these logarithmic distributions ranged from 9.2 to $14.0 \%$ ( mean $=11.8 \%, \mathrm{SD}=1.2 \%)$; the correlation of $\mathrm{CV}$ with the mean was $r=-0.05$ with $P=.68$. 
TABLE 1: Number of cases according to significance test for fit of LV myocyte breadths to log normal distribution by skewness of the logarithmically transformed breadths.

\begin{tabular}{|c|c|c|c|}
\hline \multirow[b]{2}{*}{$P$-level* } & \multirow[b]{2}{*}{ All } & \multicolumn{2}{|c|}{ Coefficient of skewness ${ }^{\dagger}$} \\
\hline & & $>-0.141$ & $<-0.140$ \\
\hline$>.8$ & 8 & 8 & 0 \\
\hline $.6-.79$ & 11 & 10 & 1 \\
\hline $.4-.59$ & 16 & 8 & 8 \\
\hline $.2-.39$ & 16 & 11 & 5 \\
\hline $.1-.19$ & 20 & 8 & 12 \\
\hline $0-.09$ & 33 & 11 & 22 \\
\hline
\end{tabular}

${ }^{*}$ Probability that the observed distribution of myocytebreadths fits the log normal form.

${ }^{\dagger}$ The mean coefficient in these 104 cases is -0.140 .

3.2. Heterogeneity of $L V$ Sites. With mean MB measured at 4 LV sites, the means can be compared in 6 pairings, testing the differences by the Tukey test. Of the 624 pairings in the pool of 104 cases, $174(28 \%)$ yielded $P<.05$, showing members of the pairs to differ significantly from each other far more often than by chance $(5 \%=31$ pairings). The numbers of pairs that differed significantly (heterogeneity) decreased with negative log normal skewness $(r=0.25$ with $P=.011$ ) indicating dissociation of significantly excessive heterogeneity from negative skewness. The $P$-value for fits of the log normal did not correlate with heterogeneity $(r=0.05$ with $P=.59)$. By components of variance analysis, the percentage of variance attributed to between sites variation averaged only $6.28 \%$ as much as error variance within sites $(\mathrm{SD}=1.00 \%$, range $=-1.6$ to $32.6 \%)$ in the 104 cases. Heterogeneity did not correlate with between-site variance across cases $(r=-0.11, P=.27)$.

3.3. Height. In the total set of 104 subjects, MB (a one dimensional quantity) was found to correlate with body length (BL, i.e., height in meters). In Figure 3, the entire body of data revealed a correlation coefficient of 0.67 . However 46 subjects entered the series because of deaths related to violence or drugs, and these are thought to offer a reasonable look at the free-living population not entering the data set because of the incidental disease that happens also to be found. Therefore, the regression equation fit only to these 46 subjects is plotted in Figure 3, $r=0.43, P<$ .001. This equation rejects an intercept term so that the appropriate equation is forced through the origin (sloping line in Figure 3):

$$
\mathrm{MB}=10.53 \times \mathrm{BL} .
$$

This equation rejects race $(P=.21)$ and sex $(P=.19)$ as offering no additionally significant correlation.

3.4. Myocyte Hypertrophy. A cutoff point for MB to define hypertrophy of myocytes was explored with logistic regression with $\mathrm{LVH}$ as response variable. The resulting equation gives $\mathrm{MB}=19.7 \mu \mathrm{m}$ as the cutoff point; above that value the mean of myocytes for that specimen can be declared

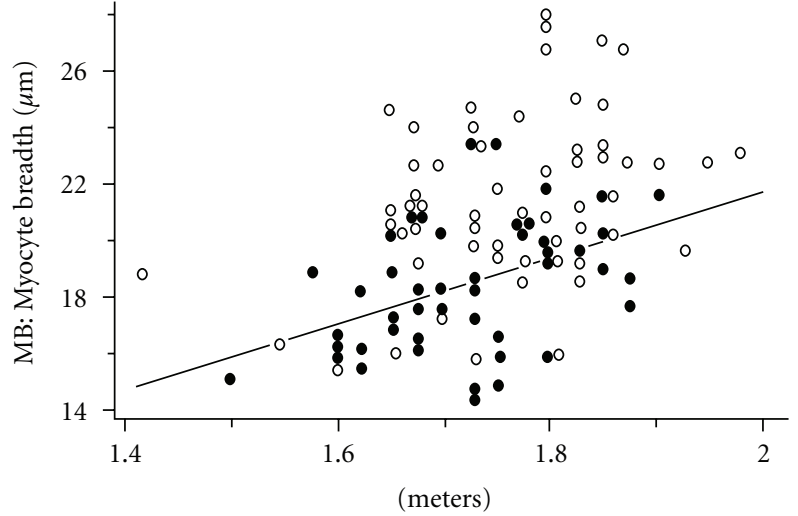

BL: body length (height)

Violent cause
- Yes
- No

Figure 3: Each symbol presents two-dimensional means of a single left ventricle. The sloping line is fit only to the dots, omitting the circles and plots (2) $(r=0.42)$. (For all 104 dots plus circles $r=$ $0.67)$.

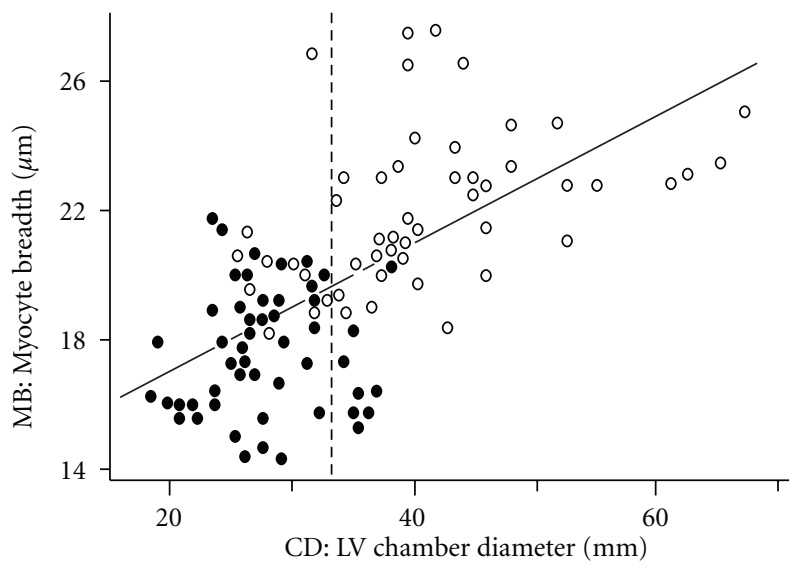

LV hypertrophy
- No
- Yes

Figure 4: Sloping line plots (3) $(r=0.63)$. Vertical dashed line plots (4), optimally separating circles from dots.

hypertrophied. Odds ratio in standard deviation units is 55.5 (10.3 to 299.3 as $95 \% \mathrm{CI}$ ).

3.5. Chamber Diameter (CD). CD (in $\mathrm{mm}$ ) correlated with $\mathrm{MB}(\mu \mathrm{m})(r=0.63)$; the regression accepts the intercept term as significant, showing lack of proportionality (sloping line in Figure 4):

$$
\mathrm{MB}=0.229 \times \mathrm{CD}+12.0
$$

(0.229 has $\mathrm{SE}=0.025,12.0$ has $\mathrm{SE}=0.91)$. 
TABLE 2: Mean myocyte breadth ( $\mu \mathrm{m})$ according to LV hypertrophy, dilatation and wall thickening (numbers of cases in parentheses).*

\begin{tabular}{lcccc}
\hline \multirow{2}{*}{ Thickened } & \multicolumn{2}{c}{ No hypertrophy } & \multicolumn{2}{c}{ Hypertrophy present } \\
& Not dilated & Dilated & Not dilated & Dilated \\
\hline No & $17.4 \mathrm{~A}$ & 16.8 & $19.4 \mathrm{~A}$ & $22.2 \mathrm{~A}$ \\
& $(38)$. & $(8)$. & $(5)$. & $(28)$. \\
Yes & $18.3 \mathrm{~A}$ & - & $21.9 \mathrm{~A}$ & $24.3 \mathrm{~B}$ \\
& $(5)$. & $(0)$. & $(5)$. & $(15)$. \\
& ANOVA & $\mathrm{F}(\mathrm{p})$ & \\
& Hypertrophy & $32.8(<.001)$ & \\
Dilatation & $11.6(.001)$ & \\
& Thickening & $2.8(.04)$ & \\
\hline
\end{tabular}

${ }^{*}$ Hypertrophy is $\mathrm{LV}$ weight $>219 \mathrm{~g}$ for men and $>199 \mathrm{~g}$ for women, dilatation is $\mathrm{LV}$ chamber diameter $>33.3 \mathrm{~mm}$, wall thickening is LV free wall thickness $>16.5 \mathrm{~mm}$; all measurements are taken in the systolic state. Means within a column that fail to share a symbol A or B differ significantly by $t$-test $(P<.05)$.

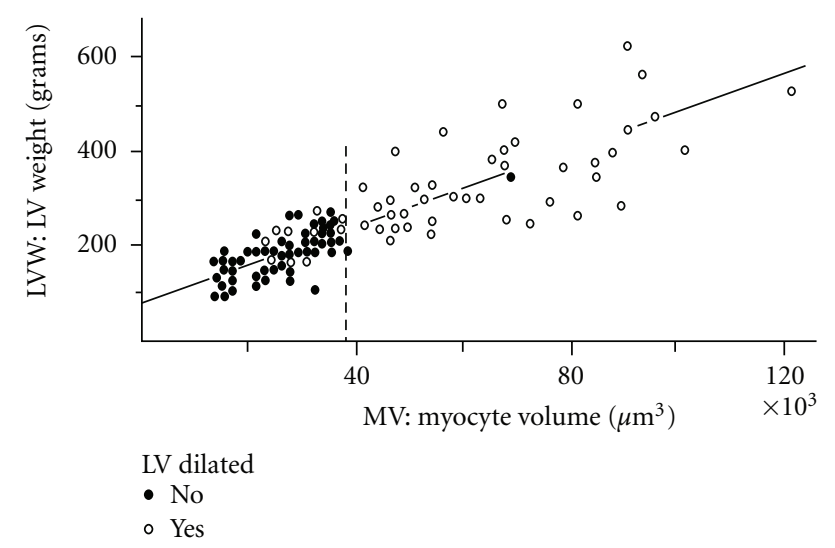

Figure 5: The sloping line plots (5) $(r=0.86)$. Vertical dashed line plots (7), optimally separating circles from dots.

The standardized version is $\mathrm{MB}=0.751 \times \mathrm{CD}$. By logistic regression, $\mathrm{LVH}$ could be predicted from $\mathrm{CD}$ :

$$
\mathrm{CD}=33.3 \text {. }
$$

This equation is plotted in Figure 4 as a dashed vertical line which optimally separates dots from circles; when $\mathrm{CD}>$ $33.3 \mathrm{~mm}$ then $\mathrm{LVH}$ is judged to be present. In standard deviation units $\mathrm{OR}=25.4(\mathrm{CI}=7.6$ to 85.1$)$.

3.6. LVW and Wall Thickness. In the 53 hearts without dilatation, a cutoff point for recognizing wall thickening was explored with logistic regression, where myocyte hypertrophy $(\mathrm{MB}>19.7 \mu \mathrm{m})$ derived in Section 3.4 was predicted using LV wall thickness, LVT. The resulting equation gives LVT $=16.5 \mu \mathrm{m}$ as the cutoff point; above this value the left ventricular free wall can be declared thickened. Odds ratio in standard deviation units is 4.21 (1.56 to $11.33 \mathrm{CI}$ ). This systolic value roughly corresponds to approximately $11.7 \mu \mathrm{m}$ in diastole.
3.7. Concentric and Eccentric Hypertrophy. Using hypertrophy without dilatation to define concentric hypertrophy, Table 2 shows this condition to display an appreciable increase in myocyte breadth compared with normal hearts, and this effect is enhanced in the presence of wall thickening. However, hypertrophied hearts with dilatation are far more numerous than those without dilatation (43 versus 10 cases), and these also express a greater degree of $\mathrm{MB}$ thickening, with the effect exceptionally strong when the ventricular free wall is thickened. The expected thinning of chamber wall as hypertrophied hearts dilate is not manifest in the group averages of Table 2 (derived from this forensic series of cases with only 4 instances of aortic stenosis contributing to the eccentric hypertrophy group).

3.8. Myocyte Volume (MV). LVW (gm, having three spacial dimensions) has a linear relationship to $\mathrm{MV}\left(\mu \mathrm{m}^{3}\right)$ described by $(r=0.86$, sloping line in Figure 5$)$

$$
\begin{gathered}
\mathrm{LVW}=3.90 \times \mathrm{MV}+72.0 \\
\left(R^{2}=.748,3.90 \text { has } \mathrm{SE}=0.23,72.0 \text { has } \mathrm{SE}=11.2\right) .
\end{gathered}
$$

The standardized equation is LVW $=0.86 \mathrm{MV}$. Logistic regression relating $\mathrm{LVH}$ to $\mathrm{MV}$ yields

$$
35,341=\mathrm{MV}
$$

$\mathrm{LVH}$ is predicted to be present at values above the cutoff, and otherwise absent, hence that cutoff defines myocyte hypertrophy $(\mathrm{OR}=931.0$ with $\mathrm{CI}=36.0$ to $>999.9$ in $\mathrm{SD}$ units). The cutoff point for using $\mathrm{MV}$ to recognize dilatation is found by logistic regression (vertical dashed line in Figure 5):

$$
37,927=\mathrm{MV}
$$

When $\mathrm{MV}>37,927 \mu \mathrm{m}^{3}$ the chamber is predicted to be dilated $(\mathrm{OR}=40.8, \mathrm{CI}=8.8$ to $187.5 \mathrm{SD}$ units $)$.

3.9. Weight. In the 70 cases with data on body weight (BW in $\mathrm{kg}$, a quantity with 3 spatial dimensions) $\mathrm{MB}=0.573 \times$ $\mathrm{BW}+14.3(r=0.551)$. The retention of an intercept term in this regression equation is inconvenient and results from comparing a three-dimensional quantity, body weight, to a one-dimensional quantity, MB. The three-dimensional quantity, MV, although limited by uncertainties in its indirect determination, correlates with BW $(r=0.50)$, and the regression equation rejects entry of an intercept term so that the appropriate equation is forced through the origin:

$$
\mathrm{MV}=435 \times \mathrm{BW}
$$

This equation rejects entry of height $(P=.92)$, body mass index $(P=.21)$, sex $(P=.61)$, and race $(P=.43)$. By logistic regression, the optimal classification of cases with myocyte hypertrophy from those without is $104.1=\mathrm{BW}(\mathrm{OR}=3.33$, $\mathrm{CI}=1.72$ to 6.45 in SD units). 
3.10. Fat and Lean Body Mass. Proxies for these quantities are introduced in methods. MV relates to these proxies with multiple correlation of $r=0.50$, thereby reproducing the value of $r=0.50$ just found for total body weight (8), as expected for the joint action of the two proxies together. The standardized regression equation for this multiple relationship is

$$
\mathrm{MV}=0.272 \times \text { height }^{2.7}+0.223 \times \mathrm{BMI}
$$

which implies that lean and fat mass proxies are of equal importance to the correlation with myocyte volume.

3.11. Mean Arterial Pressure (MAP). MAP, derived from histological renal features in 100 of the cases, was found to correlate with the measure of myocyte volume, MV ( $r=$ $0.21, P=.04)$. The intercept term is rejected so that the appropriate regression equation is forced through the origin:

$$
\mathrm{MV}=408 \times \mathrm{MAP} .
$$

By logistic regression the cutoff value for myocyte hypertrophy is MAP $=106.5 \mathrm{~mm} \mathrm{Hg}$; above this level myocyte hypertrophy becomes likely $(\mathrm{OR}=1.68$, range 1.10 to 2.58 ), and this cutoff point seems reasonable for diagnosing hypertension.

3.12. Myocyte Numbers $(M N)$. MN, total myocytes in the left ventricle, is calculated in this paragraph using formulas explained with (1), et seq. These numbers do not vary across groupings by LVW, race, sex, height, or body weight. Ventricles with myocyte hypertrophy (MV > 35, $341 \mu \mathrm{m}^{3}$ ) revealed a small deficit in cell numbers compared with normal ventricles (mean $\mathrm{MN}=5.90$ versus $6.69 \times 10^{9}$ cells, resp., SEM $=0.19$ for both means, $P<.001$ ). Similarly, dilated ventricles $(\mathrm{CD}>33.3 \mathrm{~mm})$ also showed a small deficit compared to normal $\left(\right.$ mean $=5.90$ versus $6.89 \times 10^{9}$ cells resp., $\mathrm{SEM}=0.20$ for both means, $P<.001$ ).

\section{Discussion}

Frequency distribution curves of $\mathrm{MB}$ in the present data set, in agreement with numerous published graphs [710], showed unimodal curves with conspicuous upward skewness. Fitting the log normal form in these data was seldom rejected by Shapiro-Wilk significance tests $(32 \%$ of cases rejected at $P<.1$ ), and the rejections appeared trivial to graphic inspection (Figure 2, right frame). The means of the log normal curves were uncorrelated with the coefficients of variation across the range of ventricular sizes. This is the pattern expected if myocyte hypertrophy follows an allometric growth model [26], which would indicate that the enlargement of each myocyte remains proportional to that of all others (in the absence of focal injury), a finding consistent with previous reports [7-9]. This inference might offer insights into the depressed contractility that affects myocytes as they elongate and approach failure in the dilated ventricle $[15,16]$. Houser and Margulies [15], for instance, imply that the depressed contractility in systolic heart failure, in the absence of coronary heart disease, is population wide over all myocytes, a suggestion consistent with Figure 2. Another proposal, as reviewed by Anversa et al. [27], for instance, is that tissue oxygenation is faulty and increases the vulnerability of the hypertrophied myocardium. This mechanism would presumably act selectively upon the most hypertrophied myocytes while sparing the smallest ones, a mechanism hard to reconcile with the behavior of log normal curves during hypertrophy.

The log normal distributions also seem inconsistent with the proposals of longitudinal splitting in the myocytes that are enlarged beyond the "limit of hypertrophy" [2], and of substantial cell loss to apoptosis or necrosis [16]. Those two effects might be expected to act selectively upon the most hypertrophied myocytes thereby truncating the upper range of the log normal distributions to generate a negative skewness coefficient. In the present data set, an infrequent and weak negative skewness happened as often in the smallest as in the largest ventricles thus showing no association with hypertrophy.

The lengths of minor axes in cross-sectioned myocytes, as reflected in their proxy, $\mathrm{MB}$, are not exactly random in their distribution among left ventricular samples. On average the variance between sites exhibits an excess of $6.28 \%$ above the expected value of $0 \%$ of the within-site random variance. These departures have only few and inconsistent associations with anatomic location throughout the left ventricular free wall, a finding in agreement with some authors, for example, Campbell et al. [9] "... there is no consistent pattern of regional myocyte size differences in normal mammalian hearts." These relatively small departures also do not contribute measurably to misfits of the widely ranging myocyte sizes to the log normal distributions or to their negative skewness. These departures may be of importance to cardiac function, but their significance seems negligible in the present analysis.

Zak [3] summarizes a widely held viewpoint that “... more than $90 \%$ of human postnatal growth of the heart can be accounted for by hypertrophy of the fibers present at birth." It appears that this process may continue with growth into the upper ranges of stature (Figure 3), and that the larger hearts in taller people are not manifesting physiological growth but instead are undergoing hypertrophy toward pathological boundaries. As stated by Grossman et al. [28] "in our concept, the eccentric hypertrophy by which a child's heart becomes that of an adult represents a physiologic "volume overload," and may very well utilize the same mechanisms as seen with the pathologic volume overload of valvular insufficiency." Equation (2) shows average myocyte breadth to increase in direct proportion to height, a result expected if tall persons did not receive any supplementary endowment of myocytes in infancy. These findings generate the conclusion that, in some special situations, it may be unwise to index left ventricular mass to height [26], a procedure that would artifactually discard a significant component of meaningful hypertrophy.

As the left ventricular chamber diameter (CD) is found to be increasingly dilated from one specimen to another, the 
way that myocyte breadth (MB) follows or fails to follow this change is controversial. Gerdes [12] summarizes an often encountered suggestion that, "volume overloading leads to proportionate growth in chamber diameter and wall thickness, which are reflected at the cellular level by proportionate growth of myocyte L (length) and CSA (cross-sectional area)." However, Gerdes in the same article later appears to confine the prior statement to compensated dilatation, because "... myocyte L/W (length/width) increased dramatically in humans with congestive heart failure due to ischemic and dilated cardiomyopathy." In fact, some data from Gerdes et al. [6] offer tabulations for normal and dilated failing hearts which indicate virtually no increase of myocyte breadth with dilated hypertrophy. The data in Figure 3 are generally intermediate between these extremes. Compared with the normal at $\mathrm{MB} / \mathrm{CD}=16 / 20=0.8$ (Figure 4), only one of the hypertrophied hearts fails to fall short of this value, so that a consistent proportionality of chamber diameter to myocyte breadth is unambiguously rejected in these data. The mean MB in ventricles without hypertrophy is $17.4 \mu \mathrm{m}$, and all of the hypertrophied ventricles exceed this value, so that a consistent lack of increase in $\mathrm{MB}$ with dilatation is firmly rejected. The cube of myocyte breadth, $\mathrm{MB}^{3}$, has a disappointing correlation with $\mathrm{MV}$, $r=0.59$, so that this cubic term can substitute for MV only crudely for use, perhaps, in some exploratory settings [12].

The Methods section (Elliptic eccentricity) gives a value of 1.048 for the ratio of major/minor axis in this data set. This outcome seems consistent with prior studies of autopsy materials, but is inconsistent with findings from enzymatically isolated myocytes wherein eccentricities are typically reported in the range of 2.0. There appears to be an unexplained consistent discrepancy between autopsy studies of tissue sections as compared with myocytes enzymatically isolated from surgical specimens. Although not widely entertained, one possibility is [7], “... some investigators have suggested that cardiac myocytes may assume a more flattened shape after dissociation compared with their shape in intact tissue specimens." This proposal may have some support from measurements of isolated myocyte thicknesses by confocal microscopy [14]. This uncertainty bears upon the next topic to be considered.

An adjusted value of myocyte volume used here for some limited exploratory purposes adopts the axiom that, on average, myocyte numbers are alike across the range of ventricular sizes, which allows empirical construction of (1), introducing an adjustment factor $j$. The origin of factor $j$ began from the data of Tamura et al. [13] in which the myocyte length does not quite keep up as the chamber dilates with growth and hypertrophy. This outcome implies that the laggard myocyte length must be compensated by increased myocyte numbers, by a degree of "slippage" between adjacent parallel myocytes $[2,12,13]$, or by some other means. Perhaps increased width of a few cross-wise myocytes could fill the gap. The matter remains to be clarified. The conclusions based here on estimates of MV should therefore be treated as provisional.

\section{Conclusions}

Equation (8) reports the finding that average myocyte volume can be described as proportional to total body weight. This equation rejects entry of height, as expected if lean and fat body mass contribute equally. Equation (9) tentatively supports this same conclusion. Total body weight also suffices to statistically explain the sex difference in myocyte size, the cutoff point for myocyte hypertrophy found here to be $104.1 \mathrm{~kg}$ irrespective of sex, race, height, or fatness (BMI). This surprising result derives from the little used methods of measuring myocytes as a way to diagnose hypertrophy. The conclusion is offered here as a first result of its kind and only to serve as hypothesis in need of testing.

\section{Conflict of Interests}

The authors declare that there is no conflict of interests to report.

\section{Acknowledgment}

The skilled technical input of Nicole Jackson was indispensable to this project.

\section{References}

[1] L. M. Ashley, "A determination of the diameters of ventricular myocardial fibers in man and other mammals," American Journal of Anatomy, vol. 77, no. 11, pp. 325-359, 1945.

[2] A. J. Linzbach, "Heart failure from the point of view of quantitative anatomy," The American Journal of Cardiology, vol. 5, no. 3, pp. 370-382, 1960.

[3] R. Zak, "Development and proliferative capacity of cardiac muscle cells," Circulation Research, vol. 35, supplement 2, pp. 17-26, 1974.

[4] L. H. Opi, "Mechanisms of cardiac contraction and relaxation," in Heart Disease: A Textbook of Cardiovascular Medicine, E. Braunwald, D. P. Zipes, and P. Libby, Eds., vol. 1, p. 443, W. B. Saunders, Philadelphia, Pa, USA, 2001.

[5] A. M. Gerdes, J. M. Capasso, J. Schaper et al., "Structural remodeling and mechanical dysfunction of cardiac myocytes in heart failure," Journal of Molecular and Cellular Cardiology, vol. 27, no. 3, pp. 849-865, 1995.

[6] A. M. Gerdes, S. E. Kellerman, K. B. Malec, and D. D. Schocken, "Transverse shape characteristics of cardiac myocytes from rats and humans," Cardioscience, vol. 5, no. 1, pp. 31-36, 1994.

[7] A. Zafeiridis, V. Jeevanandam, S. R. Houser, and K. B. Margulies, "Regression of cellular hypertrophy after left ventricular assist device support," Circulation, vol. 98, no. 7, pp. 656-662, 1998.

[8] B. Korecky and K. Rakusan, "Normal and hypertrophic growth of the rat heart: changes in cell dimensions and number," The American journal of physiology, vol. 234, no. 2, pp. H123-128, 1978.

[9] S. E. Campbell, B. Korecky, and K. Rakusan, "Remodeling of myocyte dimensions in hypertrophic and atrophic rat hearts," Circulation Research, vol. 68, no. 4, pp. 984-996, 1991.

[10] S. Said, T. Tamura, and A. Martin Gerdes, "Measurement of isolated myocyte volume using the Coulter models $\mathrm{Z} 2$ and 
ZM/C256: a comparison of instrument function," BioTechniques, vol. 25, no. 3, pp. 522-525, 1998.

[11] A. M. Gerdes, J. A. Moore, and J. M. Hines, "Regional differences in myocyte size in normal rat heart," Anatomical Record, vol. 215, no. 4, pp. 420-426, 1986.

[12] A. M. Gerdes, "Cardiac myocyte remodeling in hypertrophy and progression to failure," Journal of Cardiac Failure, vol. 8, no. 6, pp. S264-S268, 2002.

[13] T. Tamura, T. Onodera, S. Said, and A. M. Gerdes, "Correlation of myocyte lengthening to chamber dilation in the spontaneously hypertensive heart failure (SHHF) rat," Journal of Molecular and Cellular Cardiology, vol. 30, no. 11, pp. 21752181, 1998.

[14] A. M. Gerdes, T. Onodera, T. Tamura et al., "New method to evaluate myocyte remodeling from formalin-fixed biopsy and autopsy material," Journal of Cardiac Failure, vol. 4, no. 4, pp. 343-348, 1998.

[15] S. R. Houser and K. B. Margulies, "Is depressed myocyte contractility centrally involved in heart failure?" Circulation Research, vol. 92, no. 4, pp. 350-358, 2003.

[16] A. M. Katz, "Cardiomyopathy of overload: a major determinant of prognosis in congestive heart failure," The New England Journal of Medicine, vol. 322, no. 2, pp. 100-110, 1990.

[17] C. Grant, D. G. Greene, and I. L. Bunnell, "Left ventricular enlargement and hypertrophy. A clinical and angiocardiographic study," The American Journal of Medicine, vol. 39, no. 6, pp. 895-904, 1965.

[18] B. J. Maron and W. C. Roberts, "Quantitative analysis of cardiac muscle cell disorganization in the ventricular septum of patients with hypertrophic cardiomyopathy," Circulation, vol. 59, no. 4, pp. 689-706, 1979.

[19] K. E. Bove, D. T. Rowlands, and R. C. Scott, "Observations on the assessment of cardiac hypertrophy utilizing a chamber partition technique," Circulation, vol. 33, no. 4, pp. 558-568, 1966.

[20] R. B. Devereux, K. Wachtell, E. Gerdts et al., "Prognostic significance of left ventricular mass change during treatment of hypertension," Journal of the American Medical Association, vol. 292, no. 19, pp. 2350-2356, 2004.

[21] G. F. Bahr, G. Bloom, and U. Friberg, "Volume changes of tissues in physiological fluids during fixation in osmium tetroxide or formaldehyde and during subsequent treatment," Experimental Cell Research, vol. 12, no. 2, pp. 342-355, 1957.

[22] R. J. Siegel, K. Swan, G. Edwalds, and M. C. Fishbein, "Limitations of postmortem assessment of human coronary artery size and luminal narrowing: differential effects of tissue fixation and processing on vessels with different degrees of atherosclerosis," Journal of the American College of Cardiology, vol. 5, no. 2 I, pp. 342-346, 1985.

[23] L. E. Teichholz, T. Kreulen, M. V. Herman, and R. Gorlin, "Problems in echocardiographic volume determinations: echocardiographic-angiographic correlations in the presence or absence of asynergy," The American Journal of Cardiology, vol. 37, no. 1, pp. 7-11, 1976.

[24] R. E. Tracy, D. N. Lanjewar, K. G. Ghorpade, A. G. Valand, and S. R. Raghuwanshi, "Renovasculopathies in elderly normotensives of Bombay, India," Geriatric nephrology and urology, vol. 7, no. 2, pp. 101-109, 1997.

[25] R. E. Tracy, "The heterogeneity of vascular findings in the kidneys of patients with benign essential hypertension," Nephrology Dialysis Transplantation, vol. 14, no. 7, pp. 16341639, 1999.

[26] G. De Simone, S. R. Daniels, R. B. Devereux et al., "Left ventricular mass and body size in normotensive children and adults: assessment of allometric relations and impact of overweight," Journal of the American College of Cardiology, vol. 20, no. 5, pp. 1251-1260, 1992.

[27] P. Anversa, R. Ricci, and G. Olivetti, "Guantitative structural analysis of the myocardium duringphysiological growth and induced cardiac hypertrophy: a review," Journal of the American College of Cardiology, vol. 7, no. 5, pp. 1140-1149, 1986.

[28] W. Grossman, D. Jones, and L. P. McLaurin, "Wall stress and patterns of hypertrophy in the human left ventricle," Journal of Clinical Investigation, vol. 56, no. 1, pp. 56-64, 1975. 


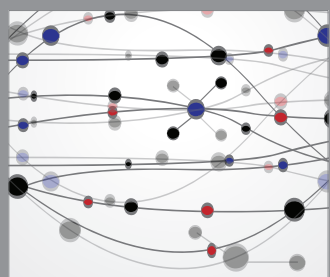

The Scientific World Journal
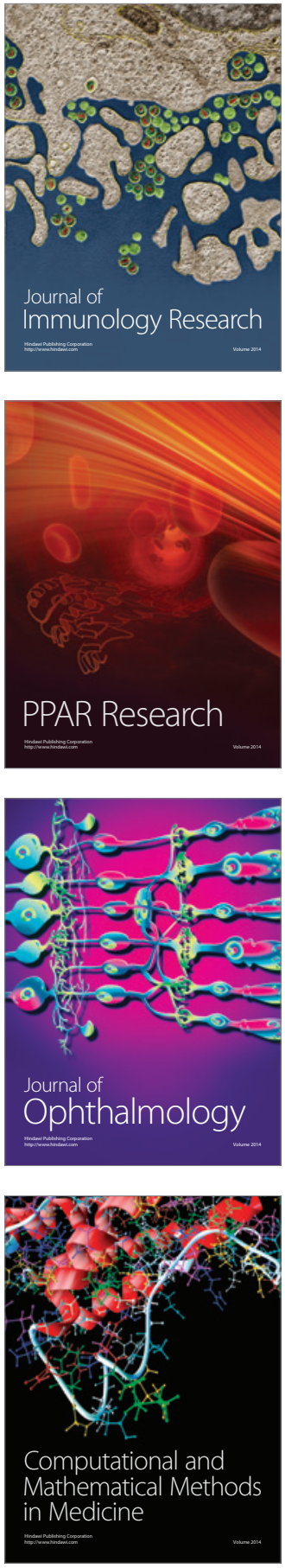

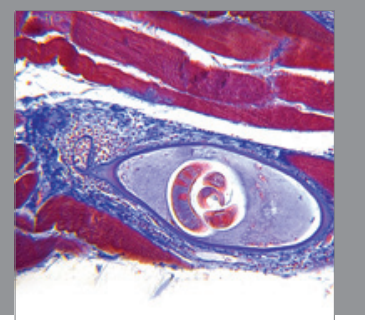

Gastroenterology

Research and Practice
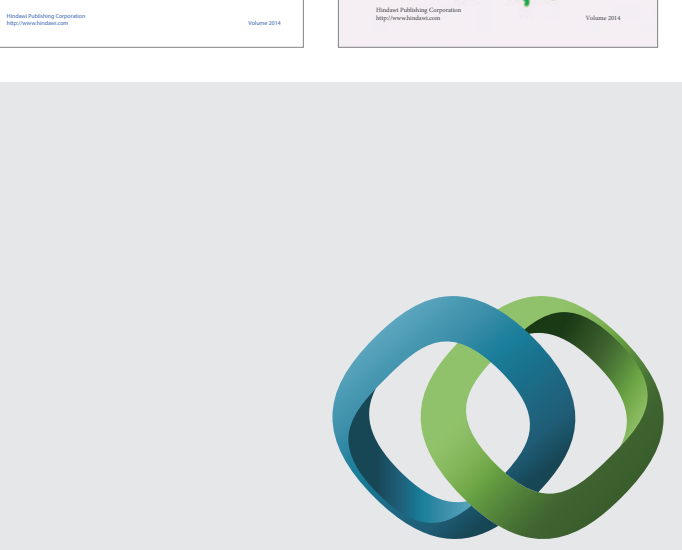

\section{Hindawi}

Submit your manuscripts at

http://www.hindawi.com
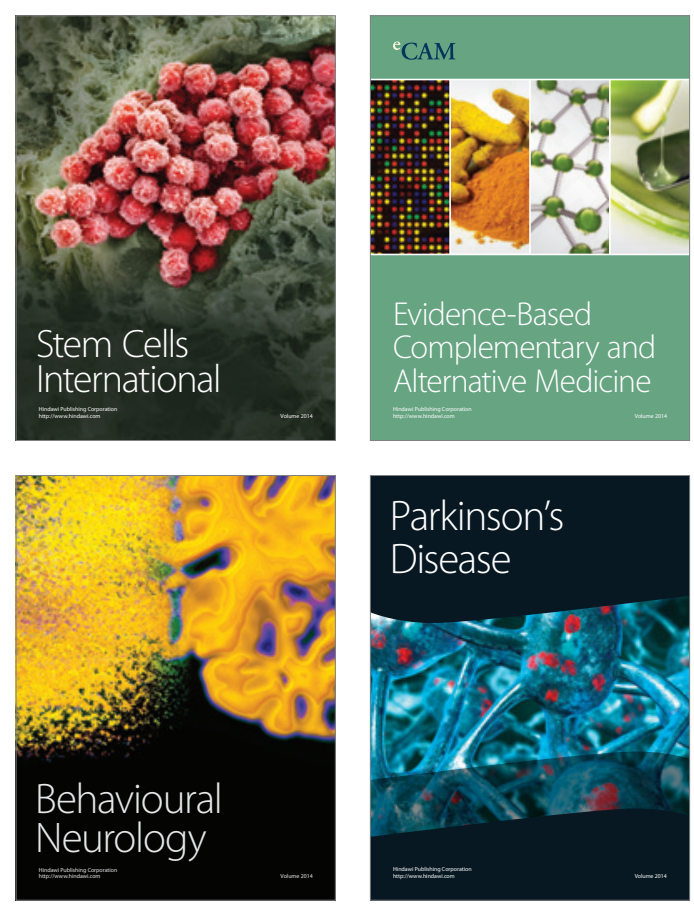

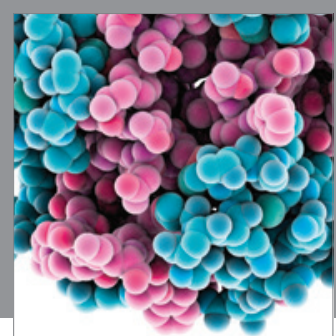

Journal of
Diabetes Research

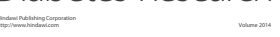

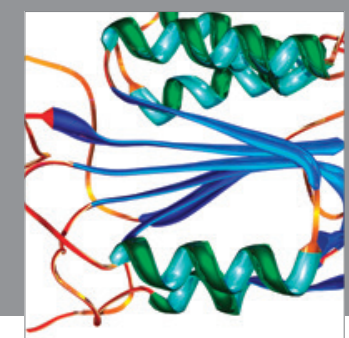

Disease Markers
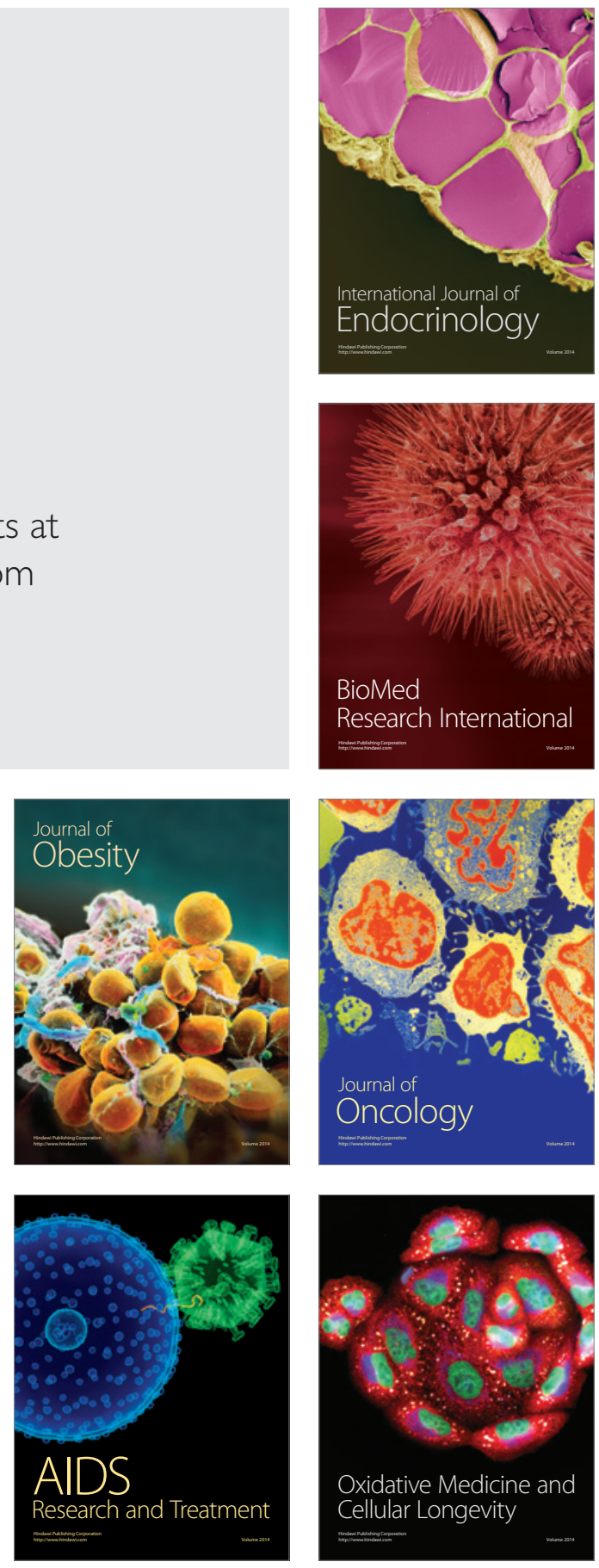